\title{
Prise en charge périnatale des nouveau-nés prématurés à la limite de la viabilité (âge gestationnel de 22 à 26 semaines complétées)
}

\author{
Les présentes recommandations ont été élaborées par une comission de spécialistes \\ et approuvées par les sociétés et fédérations concernées. La commission centrale \\ d'éthique (CCE) de I'Académie suisse des sciences médicales (ASSM) soutient ces \\ recommandations dont les modifications les plus importantes sont résumées ci-des- \\ sous.
}

\section{T. M. Berger ${ }^{a}$ \\ (responsabilité réd.), \\ V. Bernet ${ }^{a}$, \\ S. El Alama d, J.-C. Fauchèrea, \\ I. Hösli ${ }^{b}$, O. Irion ${ }^{b}$, C. Kind ${ }^{a}$, \\ B. Latal', M. Nellea, \\ R.E. Pfister ${ }^{a}$, D. Surbek ${ }^{b}$, \\ A.C. Truttmann ${ }^{a}$, J. Wisser ${ }^{b}$, \\ R. Zimmermann ${ }^{b}$ \\ (par ordre alphabétique) \\ Groupe de travail de la \\ a Société Suisse \\ de Néonatologie \\ b Société Suisse de Gynécologie et Obstétrique et de \\ l'Académie Suisse de Médecine Fœto-maternelle \\ c Société Suisse du Développe- ment \\ d Fédération suisse des sages-femmes}

\footnotetext{
* Les références se trouvent sur l'Internet www.bms.ch $\rightarrow$ Numéro actuel ou

$\rightarrow$ Archives $\rightarrow 2012 \rightarrow 4$
}

Correspondance:

Prof. Dr T. M. Berger Neonatologische und Pädiatrische Intensivpflegestation Kinderspital Luzern CH-6000 Luzern Tel. 0412053285

thomas.berger[at]ksl.ch

\section{Introduction}

Les premières recommandations relatives à la prise en charge des nouveau-nés à la limite de la viabilité en Suisse ont été publiées en 2002 [1]*. Les recommandations de groupes d'experts européens [2,3] et canadiens [4] ainsi que les directives d'éthique médicale pertinentes de l'académie suisse des sciences médicales (ASSM) $[5,6]$ avaient alors servi de base. Les révisions des recommandations provenant d'Amérique du Nord et $d^{\prime}$ Europe [7-11], les nouvelles recommandations issues d'autres pays [7-12] et les plus récentes données de mortalité et morbidité néonatales [18-22], particulièrement de la Suisse $[22,23]$, ont donné lieu à cette révision pour la Suisse.

Ces recommandations nationales sont nécessaires, puisque les prises de décision éthiques ne se basent pas seulement sur les principes éthiques reconnus, mais sont aussi influencées par des réflexions sociales, économiques et juridiques. Des données épidémiologiques de bonne qualité sur les résultats atteignables du devenir à long terme sont essentielles, et les études disponibles prouvent que ceux-ci varient considérablement entre pays [18-24]. De ce fait, les recommandations d'autres pays ne sont donc pas sans autre transférables à la Suisse. Des recommandations largement acceptées sont toutefois souhaitables afin de minimiser la variabilité des traitements entre les centres suisses qui prennent en charge des femmes enceintes à haut risque d'accouchement prématuré ainsi que des nouveau-nés prématurés à la limite de la viabilité.

Les présentes recommandations ont été élaborées par une commission de spécialistes expérimentés. Les propositions d'amendements et de modifications ont été examinées en détail lors de cinq séminaires tenus en 2009 et 2010. Les nouvelles recommandations ont été approuvées en 2011 par tous les membres du groupe de travail, par la société suisse de gynécologie et obs- tétrique (SSGO), l'académie de médecine foto-maternelle (AMFM), la fédération suisses des sages-femmes (FSS), la société suisse de pédiatrie (SSP), la société suisse de néonatologie (SSN) et la société suisse de développement (SSD). Comme déjà en 2002, la commission centrale d'éthique (CCE) de l'académie des sciences médicales (ASSM) soutient ces nouvelles recommandations. Les recommandations détaillées ont été publiées en anglais dans le Swiss Medical Weekly [25] et peuvent être lues en ligne sur www.smw.ch ou par l'intermédiaire de PubMed, ou téléchargées sous format PDF (open accès). Seules les modifications essentielles seront soulignées et présentées ici ainsi que la traduction française du résumé.

\section{Quelles sont les modifications?}

En comparaison avec la version de l'année 2002, les nouvelles recommandations contiennent des informations obstétricales basées sur l'évidence, en particulier concernant la maturation pulmonaire fotale et l'indication à la césarienne. Les anciennes recommandations basaient leurs stratégies principalement sur l'âge de gestation. Dans celles révisées, en plus de l'âge de gestation, des facteurs prénataux supplémentaires influençant de façon significative le pronostic ont été inclus dans la prise de décision (tabl. 1). La zone grise est ainsi plus étroitement définie, toutefois les frontières sont moins absolues pour permettre une prise en charge individuelle à la limite de la viabilité. L'importance de la communication est à nouveau soulignée, et le rôle des parents dans la prise de décision détaillé.

\section{Résumé}

Ces recommandations concernent la prise en charge périnatale de femmes enceintes à haut risque d'accouchement prématuré, ainsi que de nouveau-nés pré- 
maturés à la limite de la viabilité (âge gestationnel de 22 à 26 semaines complétées). Elles sont destinées aux médecins, sages-femmes, infirmières et membres d'autres professions impliqués dans cette prise en charge (afin de simplifier, la forme masculine sera dorénavant utilisée dans le texte).

La prise en charge périnatale de femmes enceintes à haut risque d'accouchement prématuré et de nouveau-nés prématurés à la limite de la viabilité (2226 semaines de grossesse complétées) doit être faite par une équipe périnatale multidisciplinaire expéri- intervenants (médecins, sages-femmes, infirmières et parents). Le but est de prendre les mesures considérées être les meilleures dans l'intérêt de l'enfant et de la femme enceinte.

La connaissance des données épidémiologiques de mortalité et morbidité par âge de gestation et l'influence sur celles-ci des facteurs prénataux (poids fœtal, sexe, maturation pulmonaire par stéroïdes, grossesse unique ou multiple), ainsi que l'application des principes d'éthique forment la base d'une prise en charge responsable. La communication entre tous les

\section{«Les décisions sont à développer dans un dialogue continu entre tous les intervenants (médecins, sages-femmes, infirmières et parents).»}

mentée. La précision limitée de la détermination de l'âge gestationnel et de l'estimation du poids fœtal, ainsi que la variabilité biologique influencent de manière décisive la prise en charge individuelle.

Les décisions à prendre pour la femme enceinte et le nouveau-né prématuré sont complexes et de longue portée. Une discussion prénatale approfondie, compréhensible et empathique de la part des néonatologues et obstétriciens, menée conjointement avec les parents, est importante pour établir une relation de confiance et offrir un soutien. Les décisions sont à développer dans un dialogue continu entre tous les acteurs joue un rôle central dans cette prise de décision.

Les membres du groupe de travail interdisciplinaire suggèrent de limiter la prise en charge des nouveau-nés prématurés d'âge gestationnel $<24$ semaines à des mesures palliatives. Les mesures obstétricales (par ex. une césarienne) pour souffrance fotale aiguë ne sont en général pas indiquées. Néanmoins, des mesures obstétricales ainsi que des soins intensifs néonataux provisoires peuvent être raisonnables dans certains cas. Elles peuvent être envisagées après la $23^{\text {e }}$ semaine complétée, lorsque plusieurs facteurs ad-

\section{Tableau 1}

Influences de facteurs prénataux additionnels (sexe, maturation pulmonaire complétée, grossesse unique ou multiple et poids de naissance) sur la probabilité de survie sans handicap sévère ou très sévère (selon Tyson et al. [20]).

\begin{tabular}{|c|c|c|c|c|c|c|c|}
\hline $\begin{array}{l}\text { Age de } \\
\text { gestation } \\
\text { (semaines) }\end{array}$ & Sexe & $\begin{array}{l}\text { Poids de } \\
\text { naissance }\end{array}$ & $\begin{array}{c}\text { Grossesse } \\
\text { unique }\end{array}$ & $\begin{array}{l}\text { Maturation } \\
\text { pulmonaire }\end{array}$ & Survie $^{1}$ & $\begin{array}{c}\text { Survie sans } \\
\text { handicap très } \\
\text { sévère }^{1,2}\end{array}$ & $\begin{array}{c}\text { Survie sans } \\
\text { handicap } \\
\text { sévère }^{1,3}\end{array}$ \\
\hline $240 / 7-246 / 7$ & $\mathrm{~m}$ & $600 \mathrm{~g}$ & non & non & $27 \%$ & $15 \%$ & $7 \%$ \\
\hline $240 / 7-246 / 7$ & $f$ & $600 \mathrm{~g}$ & non & non & $36 \%$ & $24 \%$ & $14 \%$ \\
\hline $240 / 7-246 / 7$ & $f$ & $800 \mathrm{~g}$ & non & non & $63 \%$ & $48 \%$ & $34 \%$ \\
\hline $240 / 7-246 / 7$ & $f$ & $800 \mathrm{~g}$ & oui & non & $67 \%$ & $53 \%$ & $39 \%$ \\
\hline $240 / 7-246 / 7$ & $f$ & $800 \mathrm{~g}$ & oui & oui & $82 \%$ & $71 \%$ & $57 \%$ \\
\hline
\end{tabular}

1 en pourcentage de tous les nés vivants $(n=4446)$

2 handicap très sévère (profound impairment) à l'âge de 18-22 mois:

- Bayley score $<50$ (non testable)

- level 5 pour GMF (gross motor function)

3 handicap sévère (severe impairment) à l'âge de 18-22 mois:

- PDI et/ou MDI $\leq 70$

- infirmité moteur-cérébrale (IMC) modérée à sévère

- cécité bilatérale

- surdité bilatérale nécessitant des aides auditives

Ces estimations sont basées sur des tests standardisés effecutés à 18-22 mois chez des anciens prématurées nés dans les centres du réseau NICHD entre 22 et 25 semaines de gestation et avec un poids de naissance entre 401 et $1000 \mathrm{~g}$, durant les années 1998 et 2003. Les nouveau-nés prématurées avec malformations congénitales graves et les outborn ont été exclus. 


\section{Figure 1}

Influences de facteurs additionnels positifs et rôle de l'autorité parentale lors de la pesée des options de traitement pour les enfants prématurés d'âge gestationnel de $230 / 7$ à 23 6/7 semaines.

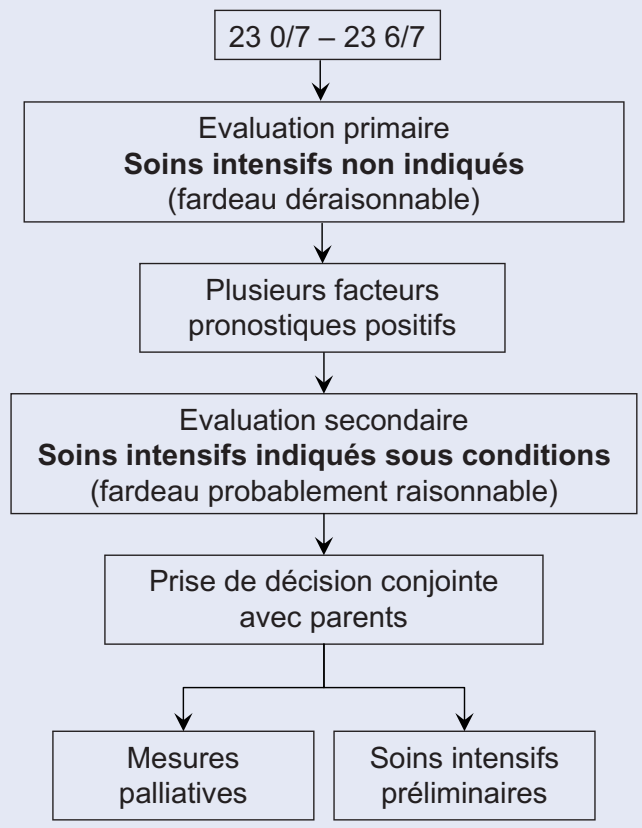

ditionnels cités ci-dessus influencent favorablement le pronostic, ou lorsque des parents soigneusement informés au préalable insistent sur l'utilisation des mesures de soins intensifs (fig. 1).

Chez le nouveau-né prématuré d'un âge de gestation entre 24 0/7-24 6/7 semaines, il peut s'avérer difficile d'évaluer si les mesures obstétricales (par exemple césarienne) et les soins intensifs néonataux sont raisonnables en vue des souffrances affligées par rapport aux chances de succès limitées. Dans cette situation, la constellation des facteurs pronostiques additionnels peut aider à la prise de décision conjointe avec les parents (fig. 2).

Pour le nouveau-né prématuré d'un âge de gestation entre $250 / 7$ et $256 / 7$ semaines, la surveillance fotale, les mesures obstétricales et les soins intensifs néonataux sont généralement indiqués. Cependant, si plusieurs facteurs pronostiques additionnels sont défavorables, une prise en charge purement palliative du nouveau-né prématuré peut être considérée dans une prise de décision partagée avec les parents (fig. 3).

Une équipe de néonatologie expérimentée doit être impliquée pour toutes les naissances après 23 0/7 semaines afin de décider individuellement et en collaboration avec les parents, si des mesures de soins intensifs sont justifiées ou si des mesures uniquement palliatives sont à prévoir (primary non-intervention). Pour cette raison, toute femme à risque d'accouche-

\section{Figure 2}

Influences de facteurs additionnels positifs respectivement négatifs et rôle de l'autorité parentale lors de la pesée des options de traitement pour les enfants prématurés d'âge gestationnel de 24 0/7 à 24 6/7 semaines.

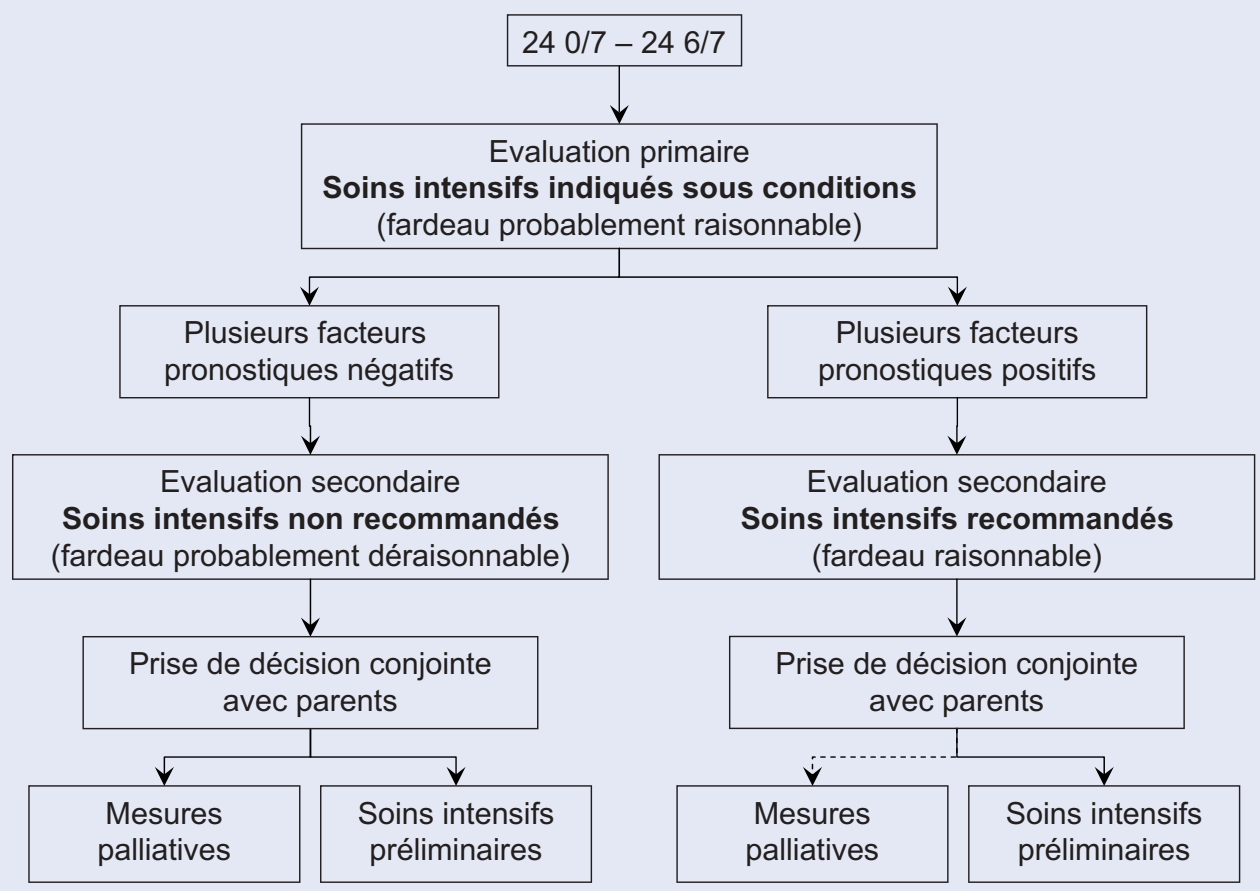


ment prématuré ou de rupture prématurée des membranes doit être transférée au plus tard dès $230 / 7$ semaines dans un centre périnatologique avec unité de soins intensifs néonataux. En cas de situations peu claires, il peut être utile d'initier la réanimation et de transférer le nouveau-né prématuré dans l'unité de

\section{«Si l'équipe soignante et les parents reconnaissent que les souffrances dues à la prise en charge thérapeutique deviennent disproportionnées en présence d'un pronostic devenu très défavorable, les mesures de soins intensifs perdent alors leur sens.»}

\section{Figure 3}

Influences de facteurs additionnels négatifs et rôle de l'autorité parentale lors de la pesée des options de traitement pour les enfants prématurés d'âge gestationnel de $250 / 7$ à $256 / 7$ semaines.

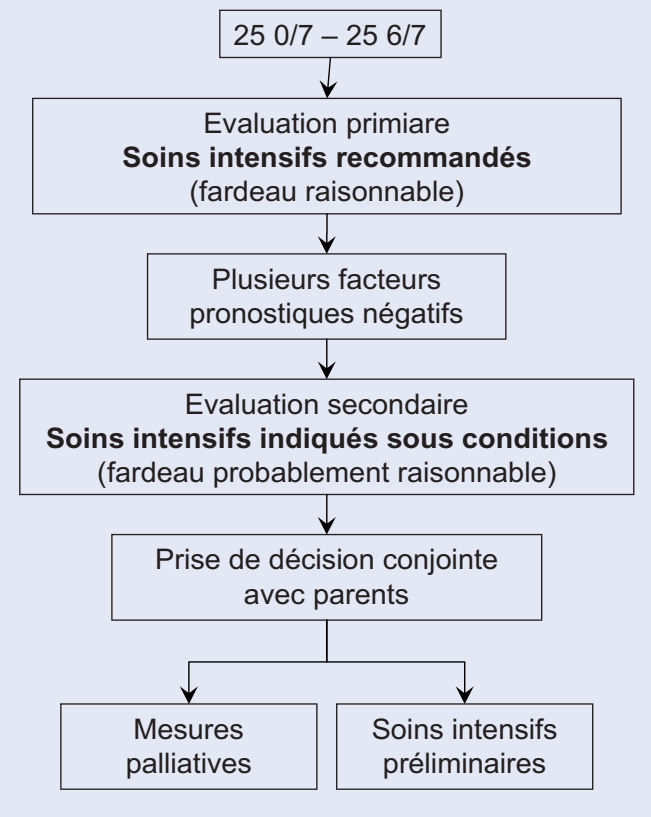

soins intensifs néonataux (provisional intensive care). L'évolution clinique et des entretiens répétés avec les parents permettront ultérieurement de clarifier si les mesures de soins intensifs doivent être poursuivies ou interrompues.

Les mesures entreprises seront poursuivies aussi longtemps que, sur la base d'évaluations fréquentes, un espoir de survie justifié existe sans que les soins intensifs mis en route deviennent déraisonnables pour le nouveau-né. Cependant, si l'équipe soignante et les parents reconnaissent que les souffrances dues à la prise en charge thérapeutique deviennent disproportionnées en présence d'un pronostic devenu très défavorable, les mesures de soins intensifs perdent alors leur sens et d'autres aspects de la prise en charge (soulagement de la douleur et de la souffrance) deviennent une priorité (redirection of care). S'il est renoncé à des interventions vitales (primaire ou secondaire), tout devra alors être mis en œuvre pour permettre à l'enfant un décès dans la dignité (comfort care) et pour soutenir les parents dans l'accompagnement de leur enfant mourant. 


\section{Réferences}

1 Berger TM, Büttiker V, Fauchère JC, Holzgreve W, Kind C, Largo R, et al. Empfehlungen zur Betreuung von Frühgeborenen an der Grenze der Lebensfähigkeit (Gestationsalter 22-26 SSW). Schweiz Ärztezeitung. 2002;83(29/30):1589-95.

2 Gee H, Dunn P, for the BAPM Executive Committee. Fetuses and newborn infants at the threshold of viability. A framework for practice. www.bapmlondon.org/publications.htm. Published 2000.

3 Pohlandt F. Frühgeburt an der Grenze der Lebensfähigkeit des Kindes. PerinatalMedizin. 1998;10:99-101.

4 Canadian Paediatric Society, Fetus and Newborn Committee, and Society of Obstetricians and Gynaecologists of Canada, Maternal-Fetal Medicine Committee. Management of the woman with threatened birth of an infant of extremely low gestational age. CMJA. 1994;151(5):547-53.

5 SAMW. Medizinisch-ethische Richtlinien für die ärztliche Betreuung sterbender und zerebral schwerst geschädigter Patienten.

Schweiz Ärztezeitung. 1995;76:1223-5.

6 SAMW. Medizinisch-ethische Richtlinien zu Grenzfragen der Intensivmedizin.

Schweiz Ärztezeitung. 1999;80:2134-8.

7 Kattwinkel J, Perlman JM, Aziz K, Colby C, Fairchild K, Gallagher J, et al. Neonatal resuscitation: 2010 American Heart Association Guidelines for Cardiopulmonary Resuscitation and Emergency Cardiovascular Care. Pediatrics. 2010;126(5):e1400-13.

8 MacDonald $H$. Perinatal care at the threshold of viability. Pediatrics. 2002;110(5):1024-7.

9 Pohlandt F. [Premature birth at the boundary of infant viability].

Z Geburtshilfe Neonatol. 2008;212(3):109-13.

10 Richmond S, Wyllie J. European Resuscitation Council Guidelines for Resuscitation 2010 Section 7. Resuscitation of babies at birth. Resuscitation. 2010;81(10):1389-99.

11 Wilkinson AR, Ahluwalia J, Cole A, Crawford D, Fyle J, Gordon A, et al. Management of babies born extremely preterm at less than 26 weeks of gestation: a framework for clinical practice at the time of birth. Arch Dis Child Fetal Neonatal Ed. 2009;94(1):F2-5.

12 Lui K, Bajuk B, Foster K, Gaston A, Kent A, Sinn J, et al. Perinatal care at the borderlines of viability: a consensus statement based on a NSW and ACT consensus workshop. Med J Aust. 2006;185(9): 495-500.

13 Miljeteig I, Markestad T, Norheim OF. Physicians' use of guidelines and attitudes to withholding and withdrawing treatment for extremely premature neonates in Norway. Acta Paediatr. 2007;96(6):825-9.
14 Moriette G, Rameix S, Azria E, Fournie A, Andrini P, Caeymaex L, et al. [Very premature births: Dilemmas and management. Part 1. Outcome of infants born before 28 weeks of postmenstrual age, and definition of a gray zone]. Arch Pediatr. 2010;17(5):518-26.

15 Moriette G, Rameix S, Azria E, Fournie A, Andrini P, Caeymaex L, et al. [Very premature births: Dilemmas and management. Second part: Ethical aspects and recommendations]. Arch Pediatr. 2010;17(5):527-39.

16 Pignotti MS, Scarselli G, Barberi I, Barni M, Bevilacqua G, Branconi F, et al. Perinatal care at an extremely low gestational age (22-25 weeks). An Italian approach: the «Carta di Firenze». Arch Dis Child Fetal Neonatal Ed. 2007;92(6):F515-6.

17 Verloove-Vanhorick SP. Management of the neonate at the limits of viability: the Dutch viewpoint. BJOG. 2006;113 Suppl 3:13-6.

18 Doyle LW, Roberts G, Anderson PJ. Outcomes at age 2 years of infants $<28$ weeks' gestational age born in Victoria in 2005. J Pediatr. 2010;156(1):49-53 e41.

19 Fellman V, Hellstrom-Westas L, Norman M, Westgren M, Kallen K, Lagercrantz H, et al. One-year survival of extremely preterm infants after active perinatal care in Sweden. JAMA. 2009;301(21):2225-33.

20 Tyson JE, Parikh NA, Langer J, Green C, Higgins RD. Intensive care for extreme prematurity - moving beyond gestational age. N Engl J Med. 2008;358(16): 1672-81.

21 Mercier CE, Dunn MS, Ferrelli KR, Howard DB, Soll RF. Neurodevelopmental Outcome of Extremely Low Birth Weight Infants from the Vermont Oxford Network: 1998-2003. Neonatology. 2009;97(4):329-38.

22 Stoll BJ, Hansen NI, Bell EF, Shankaran S, Laptook AR, Walsh MC, et al. Neonatal outcomes of extremely preterm infants from the NICHD Neonatal Research Network. Pediatrics. 2010;126(3):443-56.

23 Fischer N, Steurer MA, Adams M, Berger TM. Survival rates of extremely preterm infants (gestational age $<26$ weeks) in Switzerland: impact of the Swiss guidelines for the care of infants born at the limit of viability. Arch Dis Child Fetal Neonatal Ed. 2009;94(6):F407-13.

24 Bajwa N, Berner M, Worley S, Pfister R, Swiss Neonatal Network. Population-based age stratified morbidities of premature infants in Switzerland. Swiss Med Wkly. 2011;141:w13212.

25. Berger TM, Bernet V, El Alama S, Fauchere JC, Hosli I, Irion $\mathrm{O}$, et al. Perinatal care at the limit of viability between 22 and 26 completed weeks of gestation in Switzerland. Swiss Med Wkly. 2011;141:w13280. 